\title{
The awakening of Attneave's sleeping cat: Identification of everyday objects on the basis of straight-line versions of outlines
}

\author{
Joeri De Winter, Johan Wagemans $\uparrow$ \\ Laboratory of Experimental Psychology, University of Leuven, Tiensestraat 102, B 3000 Leuven, \\ Belgium; e-mail: johan.wagemans@psy.kuleuven.be \\ Received 21 February 2005, in revised form 31 May 2007; published online 22 February 2008
}

\begin{abstract}
Attneave (1954 Psychological Review 61 183-193) demonstrated that a line drawing of a sleeping cat can still be identified when the smoothly curved contours are replaced by straight-line segments connecting the positive maxima and negative minima of contour curvature. Using the set of line drawings by Snodgrass and Vanderwart (1980 Journal of Experimental Psychology: Human Learning and Memory 6 174-215) we made outline versions (with known curvature values along the contour) that can still be identified and that can be used to test Attneave's demonstration more systematically and more thoroughly. In five experiments (with 444 subjects in total), we tested identifiability of straight-line versions of 184 stimuli with different selections of points to be connected (using 24 to 28 subjects per stimulus per condition). Straight-line versions connecting curvature extrema were easier to identify than those based on inflections (where curvature changes sign), and those connecting salient points (determined by 161 independent subjects) were easier than those connecting midpoints. However, identification varied considerably between objects: some were almost always identifiable and others almost never, regardless of the selection criterion, whereas identifiability depended on the specific shape attributes preserved in the straight-line version of the outline in other objects. Results are discussed in relation to Attneave's original hypotheses as well as in the light of more recent theories on shape perception and object identification.
\end{abstract}

\section{Introduction}

A skilled draughtsman needs only a few well-selected line segments to depict an everyday object. Our ancestors interpreted some cracks in the rocks as representations of (parts of ) a familiar object (eg a mammoth) and added a few markings to strengthen the impression (Halverson 1992). Humans are so good at identifying objects in line drawings that it must tell something about the way the visual system extracts and processes the essential information for object identification from the more natural visual stimulation. Computer vision systems, on the other hand, have the greatest difficulties in processing new images of objects, even when they have stored several related images in their database. This glaring contrast has inspired research into the information available in line drawings (eg Koenderink and van Doorn 1982).

More than half a century ago, Attneave (1954) argued that the information about object shape is not distributed homogeneously in an image but is concentrated at contours (where the continuous distribution of spectral or grey-level values is usually the sharpest) and furthermore, that information along the contour is concentrated at points where the curvature reaches extreme values (curvature extrema). He used two demonstrations to support his intuition about the role of curvature extrema. In one demonstration, he asked participants to mark salient points along the contour of a random shape, and showed that the frequency plots were centred on the curvature extrema (see figure 1a). In a second demonstration, which has become known as Attneave's sleeping cat (see figure 1b), he created a version of a line drawing of his sleeping cat by connecting the curvature extrema by straight lines. The fact that this

T Author to whom all correspondence should be addressed. 


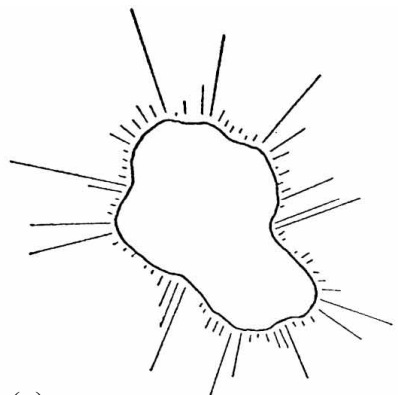

(a)

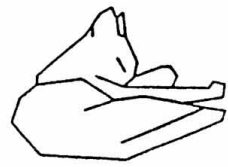

(b)

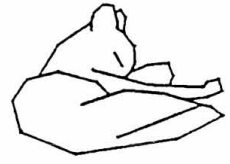

(c)

Figure 1. (a) First demonstration by Attneave (1954) of the importance of curvature extrema: people mark them as more salient points along the contour of a random shape (higher frequency is represented by a longer line). (b) Second demonstration by Attneave (1954) of the importance of curvature extrema (known as Attneave's sleeping cat). A sleeping cat can still be identified in a straight-line version connecting the curvature extrema, removing all the continuous curvature along the contour. (c) Lowe's (1985) variation in which midpoints between curvature extrema are connected by straight-line segments.

straight-line version was still easy to recognise, showed that the continuous curvature changes along the contour are not important and that the critical information about shape is located at curvature extrema.

Attneave's intuition has sparked a lot of interest in several areas of research. Resnikoff (1985) formalised the notion of information using classic measures from information theory and has provided mathematical proof of information concentration at curvature extrema. More recently, Feldman and Singh (2005) proposed an alternative quantification scheme and extended Attneave's original idea by showing that the same logic implies that negative curvature extrema (minima) must be more informative than positive curvature extrema (maxima). The reason is that all natural objects are more convex than concave and closed contours must, therefore, always turn inwards again after they have turned away from the object centre.

Attneave's demonstration about the salience of curvature extrema in random shapes (based on an unpublished study on 16 similar random shapes with 80 participants) has received further empirical support from a study on 12 silhouettes of sweet potatoes with 12 participants (Norman et al 2001). Using contour versions derived from the set of 260 line drawings of everyday objects by Snodgrass and Vanderwart (1980), we have obtained salience data from 161 participants, which appear in a separate paper (De Winter and Wagemans 2008). Attneave's anecdotal demonstration with the sleeping cat still awaits experimental confirmation when applied to other everyday objects.

There are several good reasons, both theoretical and empirical ones, for performing a more thorough study inspired by Attneave's sleeping cat. First, it is not clear how Attneave selected the salient curvature extrema to be connected by straight lines. It is highly unlikely that he did so using mathematical techniques. He probably relied on his own intuitions as a keen observer. In fact, it is quite likely that Attneave did not even start from a line drawing with continuously curved lines but immediately drew the straight-line version while he was watching his sleeping cat (see footnote 2 in Feldman and Singh 2005). Selecting curvature extrema is not a trivial matter. Mathematically, there is a local curvature extremum whenever a curvature value is surrounded by two segments of larger or smaller curvature values. When the contour is traced and its corresponding curvature values are plotted in a so-called curvature graph, all curvature singularities can be located with mathematical precision (see figure 2). It is useful to distinguish between three types of curvature singularities: positive maxima $(M+)$, negative minima $(m-)$, and inflections $(\mathrm{I})$, points where the curvature 

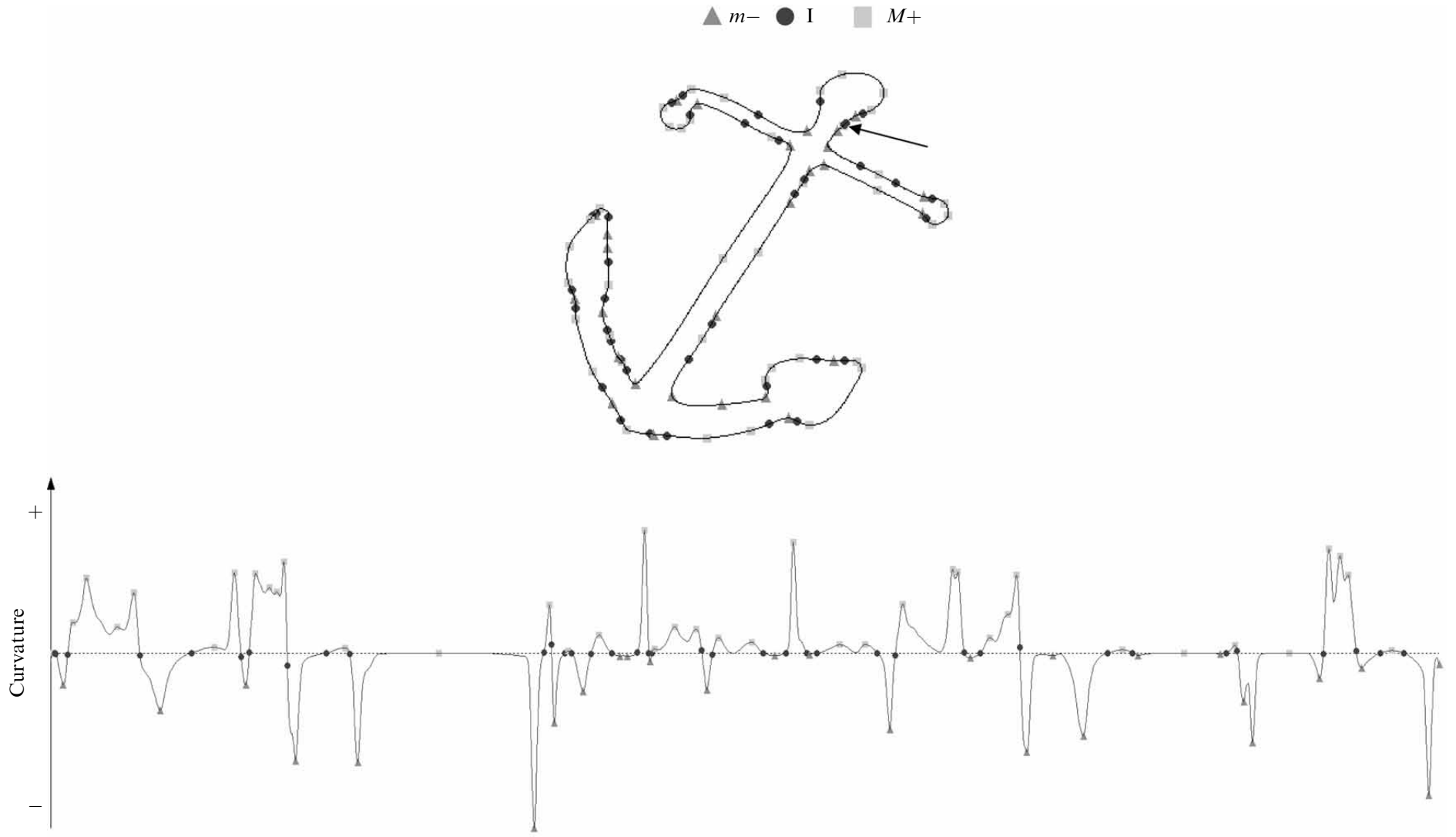

Figure 2. An example of a smoothly curved contour derived from a line drawing of an anchor by Snodgrass and Vanderwart (1980). Superimposed on the contour are the curvature singularities: local maxima of curvature or positive maxima $(M+)$ are marked by squares, local negative minima of curvature or negative minima $(m-)$ are marked by triangles, and zero crossings or inflections (I) are marked by circles. Below the outline, the curvature of the outline is plotted with the starting point indicated on the outline (the contour is traced in counterclockwise direction). The same curvature singularities are also marked on the curvature graph (with the same symbols). 
changes sign and goes through zero. The example in figure 2 shows clearly that many of the local curvature extrema are not salient (they are sometimes even located on what looks like a straight-line segment). This observation necessitates the use of a justifiable selection criterion, which Attneave did not specify. In contrast, we tested and compared several ones. The more general issue of how to define curvature (and curvature extrema) at different spatial scales and how to select the most relevant perceptual ones falls outside the scope of the present study (see Lowe 1988; Witkin 1986; and appendix 4 in De Winter and Wagemans 2006).

Second, Attneave did not use a control condition in which other points were connected by straight lines. Lowe (1985) showed that a straight-line version formed by connecting midpoints (points halfway between extrema) is also easy to identify (see figure 1c). Moreover, Biederman (1988) tested several variants of line drawings of cats (including figures $1 \mathrm{~b}$ and $1 \mathrm{c}$ ) and showed that error rates and correct naming times varied considerably, even between those that appeared easy to recognise (eg from 17\% and $1078 \mathrm{~ms}$ for figure $1 \mathrm{~b}$ and $39 \%$ and $939 \mathrm{~ms}$ for figure 1c, to about $700 \mathrm{~ms}$ and no errors for normal line drawings).

Third, in line with Lowe's sleeping cat, Kennedy and Domander (1985) showed that points halfway between corner points (midpoints) are more informative than corner points, and that points halfway between midpoints and corner points are even more informative still. They used short line segments rather than single points (for which they have been criticised by Deregowski 1986) and fragmented versions may be quite different than straight-line versions because additional processes like filling-in come into play (see also Panis et al 2008). Nevertheless, there are good mathematical reasons to believe that inflections (often close to midpoints between extrema) are perceptually important too: a change in curvature sign is easier to compute, more robust, and more stable under transformations than maxima or minima of curvature (Van Gool et al 1994) and inflections on the contour correspond to parabolic lines on the 3-D object surface, separating convex and concave surface regions (Koenderink and van Doorn 1982). In other words, comparing straight-line versions connecting curvature extrema with straight-line versions connecting other points is not just interesting for the sake of comparison; it is also theoretically meaningful.

For all these reasons, we have embarked upon a large-scale study, consisting of several experiments, in which we have tested Attneave's idea about the role of curvature extrema for object identification-as demonstrated with his sleeping cat-using much larger sets of stimuli and groups of participants. We have created silhouette and contour versions of the famous Snodgrass and Vanderwart (1980) set of line drawings of everyday objects and we have determined identification norms for them (Wagemans et al 2008; see also appendix in Wagemans et al 1998). Because we have continuous curvature values along these contours, we can also examine whether segmentations of these stimuli confirm segmentation rules like the minima rule by Hoffman and Richards (1984; see De Winter and Wagemans 2006), whether fragmented versions are indeed easier to recognise with fragments around inflections than around extrema, as suggested in the study by Kennedy and Domander (1985; see Panis et al 2008), and whether curvature extrema are more salient than other points, as suggested by Attneave's first demonstration (figure 1a) and confirmed by a more thorough study with random shapes (Norman et al 2001; see De Winter and Wagemans 2008). The study with straight-line versions which we present here is, therefore, part of a larger research program on the role of curvature singularities in shape and object perception (for an overview, see De Winter and Wagemans 2004), which provides interesting benchmark data to test several theoretical proposals, both from perceptual psychology and computer vision. 


\section{General methods}

Because all five experiments reported here belong to the same large-scale study, they share several aspects of the methods for data acquisition (eg subjects, stimuli, procedure) and data analysis (eg scoring, dependent variables, a posteriori analyses). To avoid repetition in the description of the individual experiments, we include these general aspects of the methods here, and focus on the specific details in which the methods differ between experiments below. We will also use tables to make it easier to maintain an overview of the differences and similarities between the experiments.

\subsection{Subjects}

First-year psychology students at the University of Leuven participated in all experiments in this study as a mandatory component of their curriculum. They were always naive regarding the purpose of the experiment and unfamiliar with the stimuli (we used different samples with new freshmen in each of four consecutive academic years). Depending on the number of conditions in each experiment, we used a different number of subjects to have data from 20 to 30 subjects per stimulus per condition within a reasonable time per subject (about $20 \mathrm{~min}$ ).

\subsection{Stimuli}

The stimulus set consisted of 184 straight-line versions derived from the 260 line drawings of everyday objects by Snodgrass and Vanderwart (1980). In a previous largescale study (Wagemans et al 2008; see also De Winter and Wagemans 2004), we made silhouette and contour versions of the complete Snodgrass and Vanderwart set. First, silhouettes were made by filling-in the interior surfaces in black. Their outlines were then extracted automatically and spline-fitted to obtain smooth curvature values at all points along the contour. We then determined the degree of identifiability in a group of 356 subjects. Not all of these outlines were equally easy to identify (range between $0 \%$ and $100 \%$ ), and because we expected that the identifiability would be poorer for the straight-line versions to be tested here, we selected only those that were still reasonably identifiable as a silhouette. The lower limit was set to $20 \%$, meaning that the silhouette was still named correctly by $20 \%$ of the subjects in the previous study. Based on this criterion, 66 outlines were excluded.

Some additional outlines were excluded from the remaining 194 ones for the following reasons: (i) Outlines that are completely convex have no inflections and therefore cannot be used in one of the two conditions (see below). Based on this criterion, 6 outlines were excluded (eg ball, barrel). (ii) Because of the spline-fitting procedure, some outlines had some small anomalies in the outline shape (eg small local distortions at the vertices) and they were excluded because these anomalies could have affected the straight-line versions differentially and hence our major results of interest. Based on this criterion, 3 outlines were excluded (bee, broom, needle). (iii) To be able to divide the number of stimuli into equal subgroups, we excluded one additional outline with low identifiability (telephone). These selection criteria led to a set of 184 stimuli (out of $260)$, with an average identification rate of $87.3 \%(\mathrm{SD}=19.4 \%)$. The consequence of using this subset from the Snodgrass and Vanderwart (1980) set of stimuli is that our results concern identification based on 2-D outlines of everyday 3-D objects. We assume that the original Snodgrass and Vanderwart line drawings capture the 3-D structure of their objects as well as possible but we cannot say anything about the identifiability of straight-line versions that would be derived from non-canonical views.

From the smooth contours obtained from the spline-fitted outlines, we extracted particular curvature singularities (using different selection criteria in the different experiments) and we connected them by straight lines, as Attneave (1954) may have done to create his sleeping cat. In experiments 1, 2, and 3, we compared straight-line versions connecting extrema (as Attneave did in figure 1b) with straight-line versions 
connecting inflections (often close to halfway between extrema, as Lowe did in figure 1c). In experiments 4 and 5, we started from salient points as determined empirically from another large-scale study with an independent group of 161 subjects (De Winter and Wagemans 2008). In experiment 4 , we then selected the most salient points and connected either these points by straight lines or points halfway between them (midpoints). In experiment 5, we created another variant based on the midpoints. In all of the stimuli used in the present study, we filled the interior surface of these straight-line versions with light-grey ( $75 \%$ of maximum luminance) to create silhouettes (see figures 4, 7, 8, and 9 for examples). We make these stimuli available on our website, along with their identifiability in their silhouette version as well as in the versions tested here (see http://ppw.kuleuven.be/labexppsy/johanw/wag_2D.htm).

Half of the straight-line stimuli presented to a subject were constructed with points of one type (extrema or salient points), and the other half of the stimuli with points of the complementary type (inflections or midpoints). The assignment of stimuli to conditions was always counterbalanced between subjects. The two groups of stimuli were always matched to have approximately the same average identifiability, the same number of living versus non-living objects, and the same average number of inflections.

\subsection{Procedure}

The experiments were performed in a computer classroom with 33 PCs separated by about $1 \mathrm{~m}$. There were usually multiple sessions with a maximum number of 30 subjects per session. We presented all the stimuli centred on a 17 inch CRT display at a viewing distance of approximately $0.7 \mathrm{~m}$. The display resolution was set to 1024 by 768 pixels and a refresh rate of $60 \mathrm{~Hz}$. Stimuli were all contained within a box of $640 \times$ 480 pixels (not drawn as such), for a maximum viewing angle of $16.3 \mathrm{deg}$ by $12.2 \mathrm{deg}$.

Each straight-line version was presented for a maximum of $5 \mathrm{~s}$ and then replaced by a fixation cross. Subjects were asked to identify each stimulus and subsequently input the name via the computer keyboard and click on an OK button when finished. Subjects could begin typing the name of the object as soon as they had identified the stimulus and they could type and correct as long as they wanted. If the subject clicked on $\mathrm{OK}$ in a time period shorter than $5 \mathrm{~s}$, the stimulus was removed from the screen and the next stimulus appeared. The presentation order was randomised for each subject separately and the experimenter secured silence throughout the session until he finished with the last subject.

\subsection{Scoring}

In the previous study with silhouettes and outlines (Wagemans et al 2008; see also De Winter and Wagemans 2004), we used two criteria to score the responses as correct or incorrect. With the more stringent criterion, a response was counted as correct only when the name given was the same as that listed by Snodgrass and Vanderwart (1980). With the more liberal criterion, synonyms and colloquial names that clearly indicated the same concept were also considered correct. However, remotely related names that were referring to different basic-level categories (eg "dog" for "fox" or "fly" for "bee") were not allowed. Because we used Flemish subjects in all of our experiments and Flemish has many more synonyms and colloquial names than English or Dutch (eg Severens et al 2005), we decided to use the more liberal criterion in the present study (as well as in the related ones by De Winter and Wagemans 2008 and Panis et al 2008). It is the best measure of basic-level identification. Scoring was done automatically for all names that were already in our database from the previous study. New names were scored manually by applying the same criteria (in the case of doubt, the two authors decided jointly). The database was updated with the new names (and their scoring) each time a new experiment was performed. All experiments in this study used the same scoring criteria and the results are therefore comparable across the different experiments. 


\subsection{Data analysis}

We determined the average percentages of correct identification in each condition and compared them with a $t$-test when there were only two conditions, or an analysis of variance (ANOVA) when there were more than two conditions. Because we believe that our data set may also be used as a benchmark to test specific models of object recognition (particularly computer vision or pattern recognition models), we also made available on our website the identification rates of all of the individual stimuli in all of the different versions of this study (see http://ppw.kuleuven.be/labexppsy/johanw/wag_2D.htm).

As a first step in an exploratory analysis of the factors determining identification of straight-line versions, we determined the stimulus difference between the straightline version and the original outline version for all stimuli, and correlated performance with it. The stimulus different was quantified as follows (see figure 3 for an example). First, the original silhouette was drawn in black, the straight-line version was drawn in white, and the two versions were overlaid, so that the difference in overlap in one direction was then visible in black. The area of the difference was then calculated as the number of black pixels. Next, the same procedure was repeated, but now the straight-line version was drawn in black and the original version in white and the area in this direction of the difference was also calculated. Finally, the total stimulus difference computed in this way was the sum of the two black areas remaining after applying
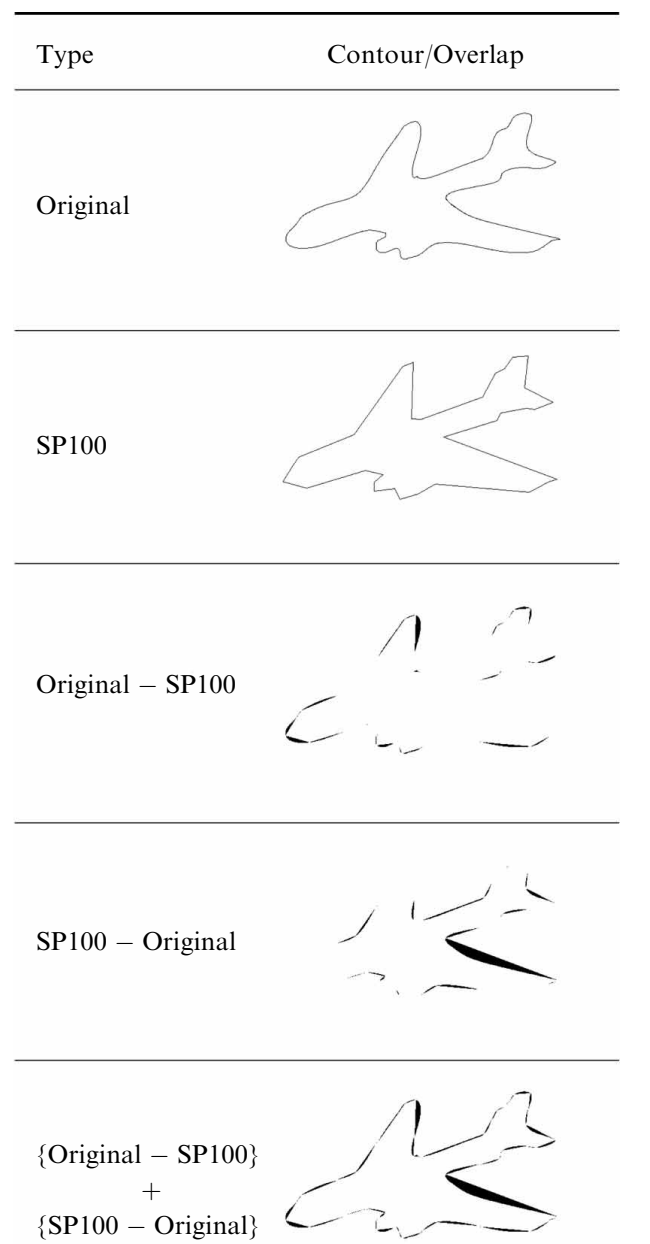

Figure 3. Stimulus difference between the straightline versions and the original outlines was quantified as the total area of the nonoverlapping parts. The three-step procedure is illustrated here for the straight-line version of the airplane in experiment 4 (connecting salient points). First, we calculated the area of the original outline (Original) minus the overlapping area of the straight-line version (SP100), resulting in the black parts shown in 'Outline - SP100'. Second, we calculated the area of the straightline version (SP100) minus the overlapping area of the original outline (Original), resulting in the black parts shown in 'SP100 - Outline'. Third, the two black areas were added. 
the overlay-and-subtract procedure in both directions. To avoid area differences in the original outlines affecting the results, this deviation measure was then divided by the original outline area. We could then correlate the relative stimulus difference for each condition with the decrease in identification rate in that condition.

We also computed other stimulus measures (contour length, number of corner points, contour length divided by the number of corner points, average length of all the straight-line segments, variability of it, etc) and correlated performance with them. However, we generally obtained weak correlations. Therefore we are not reporting them here because they did not help us in explaining the differences in identification rate. In section 8 we include some of our observations from these exploratory analyses, as an illustration of the potential of this approach. All our stimuli have also been made available on our website (both as bitmaps and as pixel files) so that anyone with particular ideas about relevant stimulus variables determining identification can test them on our benchmark data sets (see http://ppw.kuleuven.be/labexppsy/johanw/wag_2D.htm).

\section{Experiment 1: Curvature singularities-one extremum per lob}

\subsection{Introduction}

In the first three experiments, curvature singularities were corner points connected by straight-line segments. They differed only in the way the curvature singularities were selected. In experiment 1 , we selected only one curvature extremum for each segment of the contour with a particular sign of curvature: one maximum $(M+)$ per segment of positive curvature and one minimum $(m-)$ per segment of negative curvature.

\subsection{Methods}

The aspects of the methods common to all of the experiments have been reported in section 2, above. Here, we describe only the specific aspects of the methods that are unique to this experiment (see table 1 for an overview).

Table 1. Methodological details of the five experiments.

\begin{tabular}{lcclll}
\hline & \multicolumn{2}{c}{ Experiment } & & & \\
\cline { 2 - 6 } & 1 & 2 & 3 & 4 & 5 \\
\hline Number of subjects & 56 & 50 & 206 & 108 & 24 \\
Number of stimuli & 184 & 184 & 184 & 184 & 142 \\
Stimulus conditions & E vs I & E vs I & E vs I & SP100 vs MP100 & MPT \\
Number of stimuli per subject & 184 & 184 & 46 & 184 & 142 \\
Number of subjects & 28 & 25 & 26 & 27 & 24 \\
$\quad$ per stimulus per condition & & & & & \\
\hline
\end{tabular}

3.2.1 Subjects. 56 participants (13 male, 43 female, mean age 19.3 years) were tested in two sessions with a maximum number of 30 participants in each session.

3.2.2 Stimuli. The two conditions in this experiment consisted of straight-line versions connecting either curvature extrema (E) or inflections (I). Each outline contour in our set of stimuli had more extrema than inflections. To make sure that the number of points to be connected was equal in both conditions, we used the following criterion to select extrema. Within a fragment of positive or negative curvature (called here a lob), we selected the extremum with the highest absolute curvature. This automatically results in an equal number of extrema and inflections per outline, because each lob with positive curvature is followed by a lob of negative curvature (and vice versa), with an inflection point inbetween. The mean number of selected points across stimuli was 29.7 $($ median $=24.5)$. Stimulus examples are shown in figures 4,7 , and 8 . 


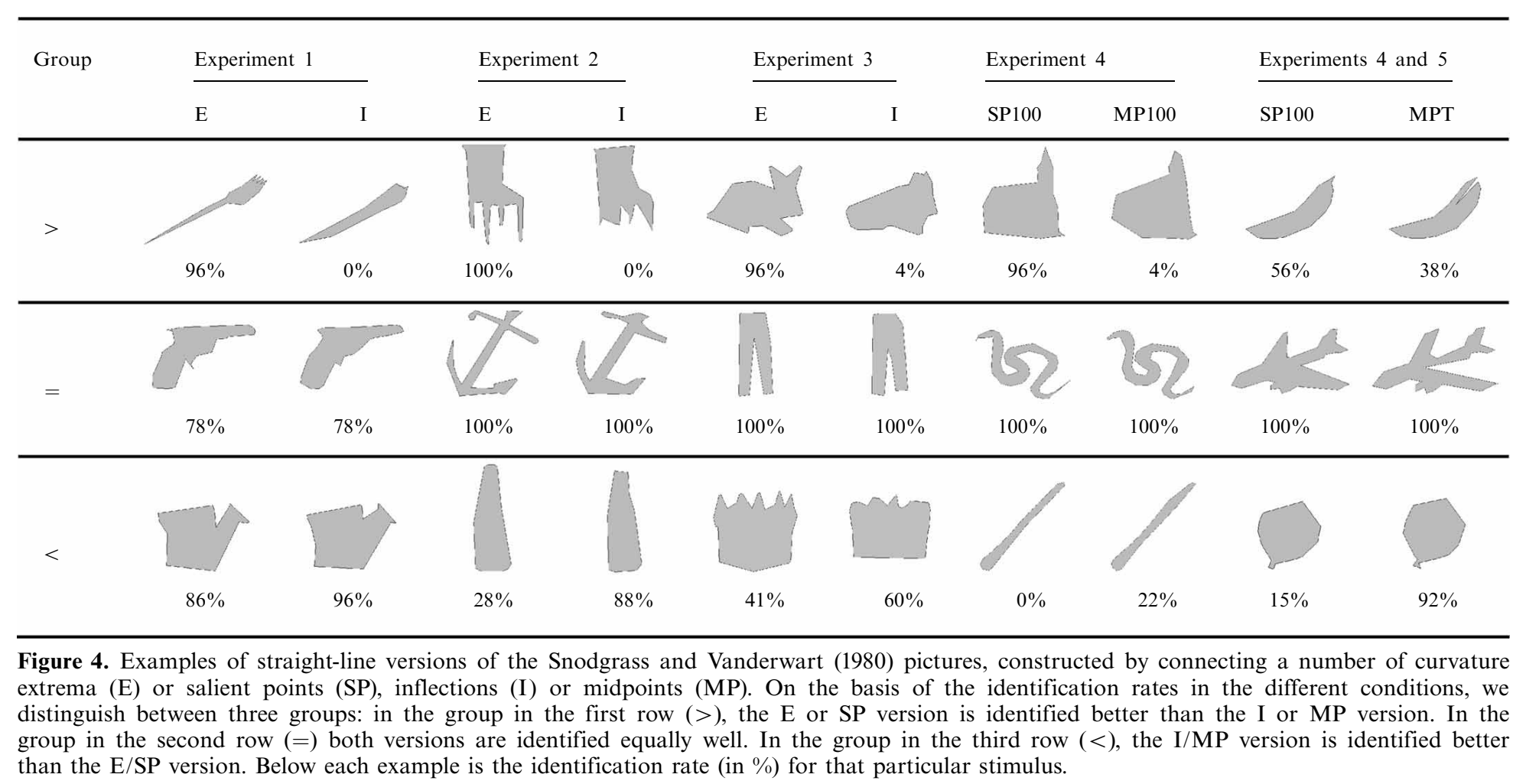


3.2.3 Procedure. Each subject received all 184 stimuli, half of them in E version and half in I version (stimulus assignment was counterbalanced across subjects). Each stimulus was thus presented to 28 subjects per condition.

\subsection{Results and discussion}

The most important identification results are tabulated: separately per condition in table 2 (see over), and comparatively between conditions in table 3. Some stimulus measures that might correlate with identifiability are included in table 4 . The distribution of identification rate in the two conditions is plotted in figure 5a.

Identification was still reasonable or good for the straight-line versions connecting the extrema (E versions), but it was rather poor for the straight-line versions connecting the inflections (I versions). Mean and median identifications were $46.4 \%$ and $41.1 \%$ for E versions and $20.4 \%$ and $3.6 \%$ for I versions (see table 2). A $t$-test on the means revealed a large statistical difference between the two conditions: $t_{366}=7.28$, $p<0.001$. The fact that $\mathrm{E}$ versions can still be identified in many cases (eg 50 are within $10 \%$ of the identification rate of the original outlines) supports Attneave's (1954) idea that continuous curvature changes are often not needed. Moreover, the fact that E versions are easier to identify than I versions confirms that Attneave's version of the sleeping cat (figure 1b) was more representative of everyday objects than Lowe's version (figure 1c). However, there was a considerable range of identification performance across the stimuli (see figure 5a): there are relatively more E versions with high identification rates $(\mathrm{eg}>70 \%)$ than I versions, and more I versions with lower identification rates $(\mathrm{eg}<30 \%)$ than E versions, but in both versions identifiability is distributed across the whole range from $0 \%$ to $100 \%$. This stimulus variability may be informative about stimulus variables affecting identifiability.

As a point of departure for an exploratory analysis of stimulus differences leading to these identification differences, we distinguished three groups of stimuli (see table 3): (i) those in which the identification rates were in line with the average difference $(E>I)$, (ii) those with no difference $(E=I)$, and (iii) those in which the difference was in the opposite direction $(E<I)$. Examples of each group are given in figure 4. The first group was clearly the largest (127 stimuli or $69.0 \%$ of the stimuli), and the mean and median difference in identification here was $38.6 \%$ and $35.7 \%$, respectively. The second group consisted of 45 stimuli $(24.5 \%$ of the stimuli), 41 of which were not identified at all ( $0 \%$ identification in both conditions). Many of the

Table 3. Overview of the comparative results of the five experiments.

\begin{tabular}{|c|c|c|c|c|c|c|}
\hline & \multicolumn{6}{|c|}{ Experiment } \\
\hline & 1 & 2 & 3 & $4(100 \%)$ & $4(75 \%)$ & 5 \\
\hline $\begin{array}{l}\text { Number of stimuli in first group } \\
(E>\text { I or } S P>M P)\end{array}$ & 127 & 154 & 166 & 163 & 152 & 73 \\
\hline Mean difference in first group & 38.6 & 12.5 & 45.6 & 39.6 & 44.9 & 22.3 \\
\hline Q1 of difference in first group & 17.9 & 20.0 & 20.0 & 15.0 & 18.5 & 8.3 \\
\hline Median difference in first group & 35.7 & 46.0 & 39.9 & 33.0 & 44.4 & 20.8 \\
\hline Q3 of difference in first group & 57.1 & 76.0 & 73.1 & 59.0 & 66.7 & 29.6 \\
\hline $\begin{array}{l}\text { Number of stimuli in second group } \\
(E=1 \text { or } S P=M P)\end{array}$ & 45 & 19 & 11 & 14 & 24 & 21 \\
\hline $\begin{array}{l}\text { Number of stimuli in third group } \\
(\mathrm{E}<1 \text { or } \mathrm{SP}<\mathrm{MP})\end{array}$ & 12 & 11 & 7 & 7 & 8 & 48 \\
\hline Mean difference in third group & -6.5 & -15.3 & -11.5 & -14.8 & -10.2 & -16.2 \\
\hline Q1 of difference in third group & -12.5 & -16.0 & -14.9 & -17.0 & -12.0 & -23.4 \\
\hline Median difference in third group & -3.6 & -12.0 & -10.2 & -15.0 & -9.3 & -10.6 \\
\hline Q3 of difference in third group & -3.6 & -6.0 & -4.1 & -13.0 & -6.5 & -4.6 \\
\hline
\end{tabular}


Table 2. Overview of the identification results (in \%) of the five experiments.

\begin{tabular}{|c|c|c|c|c|c|c|c|c|c|c|c|c|c|}
\hline \multirow[t]{3}{*}{ Identification rate } & \multicolumn{13}{|c|}{ Experimental condition } \\
\hline & \multirow{2}{*}{$\begin{array}{l}\text { Full } \\
184\end{array}$} & \multicolumn{2}{|c|}{ Experiment 1} & \multicolumn{2}{|c|}{ Experiment 2} & \multicolumn{2}{|c|}{ Experiment 3} & \multicolumn{4}{|c|}{ Experiment 4} & \multirow{2}{*}{$\frac{\text { Experiment } 5}{\text { MPT }}$} & \multirow{2}{*}{$\begin{array}{l}\text { Full } \\
142\end{array}$} \\
\hline & & $\mathrm{E}$ & I & $\mathrm{E}$ & I & $\mathrm{E}$ & I & SP100 & MP100 & SP75 & MP75 & & \\
\hline Mean & 87.3 & 46.4 & 20.4 & 53.4 & 14.2 & 60.7 & 20.0 & 68.9 & 34.4 & 54.7 & 18.0 & 60.2 & 86.9 \\
\hline SD & 19.4 & 39.3 & 28.1 & 36.4 & 23.7 & 35.3 & 29.1 & 34.1 & 32.9 & 37.8 & 25.7 & 33.0 & 19.8 \\
\hline $\mathrm{Q}^{\mathrm{a}}$ & 82.6 & 3.6 & 0.0 & 16.0 & 0.0 & 24.8 & 0.0 & 39.8 & 3.7 & 18.5 & 0.0 & 29.2 & 81.7 \\
\hline Median ${ }^{\mathrm{a}}$ & 97.7 & 41.1 & 3.6 & 50.0 & 0.0 & 71.7 & 3.8 & 85.2 & 25.9 & 59.3 & 3.7 & 62.5 & 98.3 \\
\hline $\mathrm{Q}^{\mathrm{a}}$ & 100.0 & 86.6 & 35.7 & 92.0 & 16.0 & 92.7 & 29.0 & 100.0 & 59.3 & 96.3 & 29.6 & 91.7 & 100.0 \\
\hline$\# 0 \%$ b & 0 & 45 & 78 & 18 & 96 & 9 & 82 & 7 & 33 & 21 & 75 & 6 & 0 \\
\hline$\# 100 \%{ }^{\mathrm{b}}$ & 59 & 20 & 0 & 26 & 1 & 30 & 2 & 52 & 5 & 32 & 0 & 22 & 48 \\
\hline \#within $10 \%{ }^{\mathrm{c}}$ & - & 50 & 11 & 56 & 2 & 65 & 13 & 89 & 15 & 63 & 1 & 53 & - \\
\hline
\end{tabular}

${ }^{\mathrm{a}} \mathrm{Q} 1=$ first quartile $(25 \%$ point of the distribution $), \mathrm{Q} 3=$ third quartile $(75 \%$ point $)$, and median $=$ second quartile $(50 \%$ point $)$.

${ }^{\mathrm{b}} \# 0 \%=$ the number of stimuli with $0 \%$ identification and $\# 100 \%=$ the number of stimuli with $100 \%$ identification.

${ }^{\mathrm{c}}$ \#within $10 \%=$ the number of stimuli that are identified more or less as the same as the original version $( \pm 10 \%)$.

Table 4. Overview of the correlational measures of the five experiments.

\begin{tabular}{|c|c|c|c|c|c|c|c|c|c|c|c|c|c|c|}
\hline & \multirow{2}{*}{$\begin{array}{l}\text { Full } \\
M+\end{array}$} & \multirow{2}{*}{$\begin{array}{l}\text { Full } \\
m-\end{array}$} & \multirow{2}{*}{$\begin{array}{l}\text { Full } \\
\text { I }\end{array}$} & \multicolumn{2}{|c|}{ Experiment 1} & \multicolumn{2}{|c|}{ Experiment 2} & \multicolumn{2}{|c|}{ Experiment 3} & \multicolumn{4}{|c|}{ Experiment 4} & \multirow{2}{*}{$\begin{array}{l}\text { Experiment } 5 \\
\text { MPT }\end{array}$} \\
\hline & & & & E & I & E & I & E & I & SP100 & MP100 & SP75 & MP75 & \\
\hline \multicolumn{15}{|c|}{ Number of points } \\
\hline mean & $\begin{array}{r}19.1 \\
9.0\end{array}$ & $\begin{array}{l}25.2 \\
14.0\end{array}$ & $\begin{array}{l}30.0 \\
16.0\end{array}$ & $\begin{array}{l}29.7 \\
24.5\end{array}$ & $\begin{array}{l}29.7 \\
24.5\end{array}$ & $\begin{array}{l}27.4 \\
24.0\end{array}$ & $\begin{array}{l}24.9 \\
200\end{array}$ & $\begin{array}{l}24.2 \\
220\end{array}$ & $\begin{array}{l}18.8 \\
170\end{array}$ & $\begin{array}{l}27.6 \\
240\end{array}$ & $\begin{array}{l}27.6 \\
240\end{array}$ & 20.6 & 20.6 & 17.7 \\
\hline median & $\begin{array}{l}9.0 \\
0.061\end{array}$ & $\begin{array}{c}14.0 \\
0.089\end{array}$ & $\begin{array}{c}16.0 \\
0.049\end{array}$ & $\begin{array}{c}24.5 \\
0.47\end{array}$ & $\begin{array}{c}24.5 \\
0.48\end{array}$ & & & $\begin{array}{c}22.0 \\
0.18\end{array}$ & $\begin{array}{c}17.0 \\
0.29\end{array}$ & $\begin{array}{c}24.0 \\
0.30\end{array}$ & $\begin{array}{c}24.0 \\
039\end{array}$ & 18.0 & 18.0 & 14.0 \\
\hline$r$ & 0.061 & 0.089 & 0.049 & 0.47 & 0.48 & 0.21 & 0.33 & 0.18 & 0.29 & 0.30 & 0.39 & 0.27 & 0.37 & 0.25 \\
\hline$p$ & 0.413 & 0.231 & 0.505 & 0.000 & 0.000 & 0.004 & 0.000 & 0.017 & 0.000 & 0.000 & 0.000 & 0.000 & 0.000 & 0.003 \\
\hline \multicolumn{15}{|c|}{ Area difference/pixels } \\
\hline mean & & & & 8616 & 11249 & 7869 & 13283 & 3765 & 11457 & 2906 & 5154 & 6626 & 8030 & 2445 \\
\hline median & & & & 6197 & 9078 & 5913 & 11296 & 3238 & 9394 & 2639 & 4665 & 5034 & 6785 & 2142 \\
\hline$r$ & & & & 0.53 & 0.35 & 0.28 & 0.26 & 0.14 & 0.33 & -0.09 & -0.11 & 0.24 & 0.02 & 0.08 \\
\hline$p$ & & & & 0.000 & 0.000 & 0.000 & 0.000 & 0.066 & 0.000 & 0.360 & 0.150 & 0.000 & 0.812 & 0.200 \\
\hline
\end{tabular}



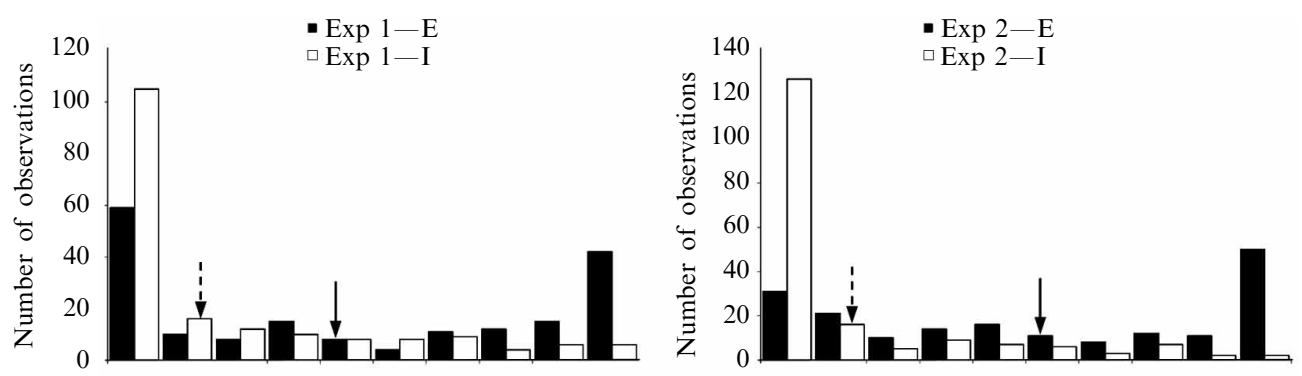

(a)

(b)
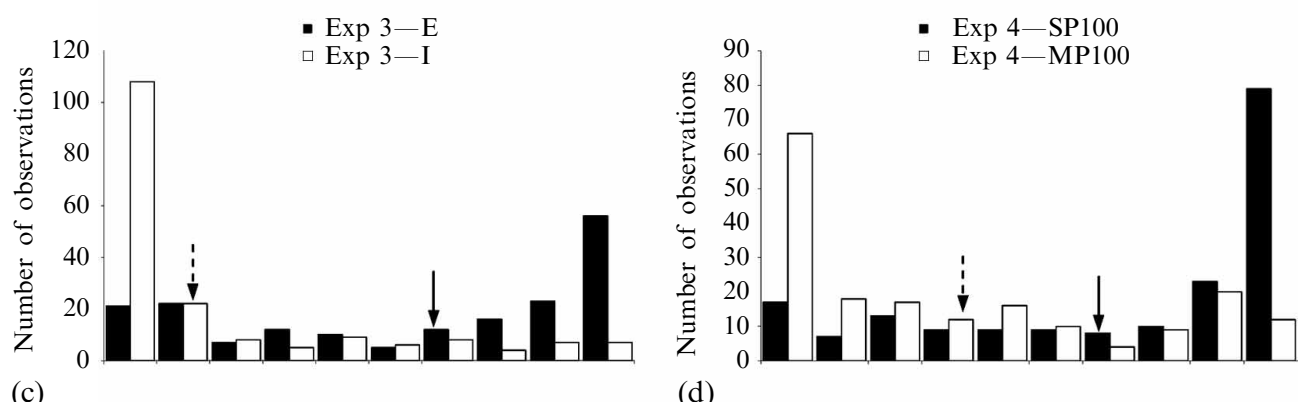

(d)
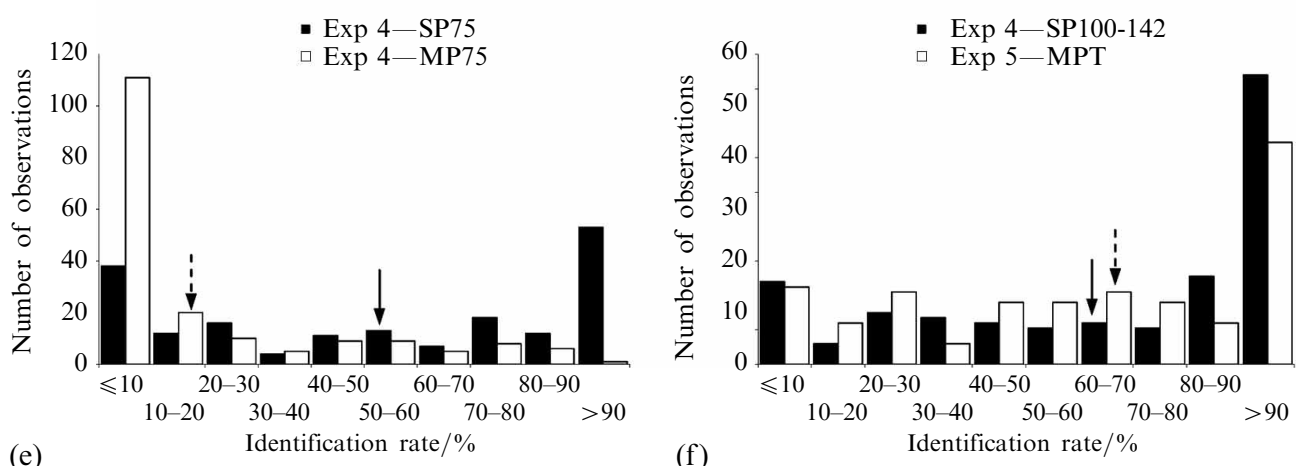

Figure 5. Distribution of the identification rates in all of the experimental conditions. In each panel, the number of observations in each of the $10 \%$ bins is indicated, separately for the E/SP versions (in black) and the I/MP versions (in white). The location of the average identification rate along the $x$-axis is indicated by an arrow (solid for the E/SP versions and dashed for the I/MP versions). (a) Experiment 1. (b) Experiment 2. (c) Experiment 3. (d) Experiment 4; 100\% versions. (e) Experiment 5, 75\% versions. (f) Comparison between the SP100-142 of experiment 4 (see text for more details) and MPT versions of experiment 5. In all of the experiments, the distribution for $\mathrm{E} / \mathrm{SP}$ versions is skewed more to the right (higher identifiability), whereas the distribution for I/MP versions is skewed more to the left (lower identifiability).

objects in this category have large round sections in the outline (eg 'apple' in figure 6a). Owing to our selection procedure in this experiment (only one extremum per lob), the straight-line versions have only a small number of straight-line segments with large parts of the shapes chopped off and sharp corners in both the $\mathrm{E}$ and the I versions. In the last group, with only 12 stimuli $(6.5 \%)$, the I versions were easier to identify than the $\mathrm{E}$ versions, but the difference was usually small (mean $=-6.5 \%$, median $=-3.6 \%$ ). Sometimes the identification difference seems to be based on some 


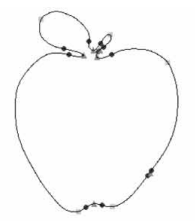

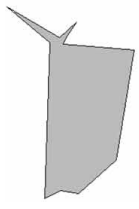

$\mathrm{E}-0 \%$

(a)

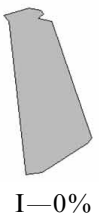

$\mathrm{I}-0 \%$
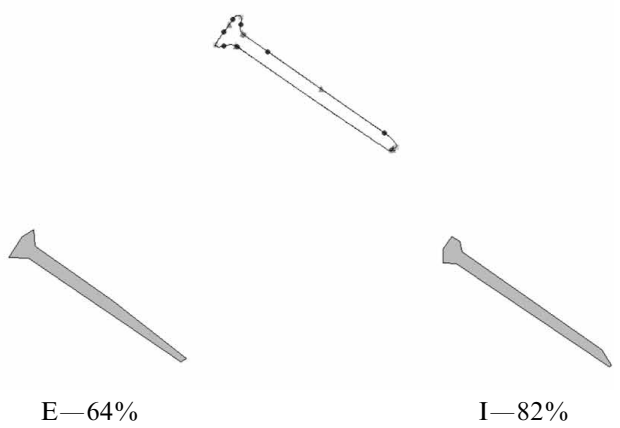

(b)

Figure 6. Examples of straight-line versions derived from outlines. (a) Outline of an apple with curvature singularities marked as in figure 2. Below the outline are the straight-line versions connecting extrema (E) or inflections (I), shown left and right, respectively. None of these versions can be identified because large round parts are chopped off and replaced by a single straight-line segment in both cases. (b) Outline of a nail with curvature singularities marked as in figure 2. Below the outline are the straight-line versions. The I version appeared somewhat easier to identify than the E version (probably based on a local detail such as the tip of the nail). The identification rate is mentioned below each stimulus.

stimulus feature that may be diagnostic and that happens to be preserved better in the I version than in the E version (eg 'nail' in figure 6b). We will return to the possible shape differences underlying the variability in identification rate in the straight-line versions in section 8 .

We explored to what extent these identification differences can be explained by a simple factor (see table 4): stimulus difference between the straight-line version and the original outline (see section 2 for computational details). Correlation between the magnitude of the stimulus difference (sum of the overlap differences relative to the original silhouette area) and the identification difference (identification rate of the original silhouette minus identification rate of the straight-line version) was reasonably high: $r=0.53$ for the E condition $(p<0.001)$ and $r=0.35$ for the I condition $(p<0.001)$. We will return to this in section 8 .

\section{Experiment 2: Curvature singularities-strongest extrema}

\subsection{Introduction}

In this experiment, we have dropped the restriction that each contour segment must deliver one extremum to be connected by straight-line segments. Here, we have just taken the strongest extrema for each outline, regardless of where they are located along the contour. The number of extrema to be selected (and the corresponding number of inflections) is determined by the number of salient points used in experiment 4. This enables a direct comparison between a mathematical selection criterion (extrema with highest absolute curvature) and a perceptual one (visual salience as judged by a large group of independent subjects).

\subsection{Methods}

Only aspects of the methods that are unique to this experiment are described here (see also section 2 and table 1).

4.2.1 Subjects. 50 participants (10 male, 40 female, mean age 19.1 years) were tested in two sessions with a maximum number of 30 participants in each session. 
4.2.2 Stimuli. On the basis of the results of an independent study, in which 161 subjects were asked to mark salient points on the contours of all 260 stimuli (De Winter and Wagemans 2008; see also De Winter and Wagemans 2004), we selected the most salient points to be connected with straight-line segments in experiment 4 (see below). For all stimuli in experiment 2, we selected curvature extrema with the highest absolute curvature until we had the same number of points as in experiment 4 (mean $=27.4$ points). In contrast to experiment 1 , where all inflections were used, the selection of inflections was less obvious in this experiment because there is no simple criterion to determine which ones are most important. To make the selection comparable to that of the extrema, we ranked all inflections by the average absolute curvature of the two selected neighbouring extrema, and selected those with the highest values until the criterion number was reached as closely as possible (there were a few cases where not enough were available; mean $=24.9$ points). As in all the other experiments, the selected points (either E or I) were then connected by straight-line segments to have E versions and I versions of all 184 stimuli (see figures 4, 7, and 8).

4.2.3 Procedure. Each subject received all 184 stimuli (half in E version and half in I version, with stimulus assignment counterbalanced across subjects); each stimulus was thus presented to 25 subjects per condition.

\subsection{Results and discussion}

For the most important results, see tables 2, 3, and 4 and figure 5b. As in experiment 1, identification was still reasonable to good for the E versions, whereas it was poor for the I versions: mean and median identifications were $53.4 \%$ and $50.0 \%$ for E versions and $14.2 \%$ and $0.0 \%$ for I versions. A $t$-test on the averages revealed a large statistical difference between the two conditions $\left(t_{366}=12.25, p<0.001\right)$. The difference between $\mathrm{E}$ and I versions has become even bigger than in experiment 1. This increased difference may be due to the average number of selected extrema and inflections, which differ slightly here (27.4 or 24.9 , respectively), whereas they were equal in experiment 1 (29.7). In addition, it may be due to some properties of the selected points: first, the selection of extrema was probably better in this experiment because the largest extrema were now selected (regardless of their location along the contour), and, second, the selection of inflections was probably worse in this experiment (because their selection was derived from the curvature values of the neighbouring extrema rather than being evenly distributed along the contour as in experiment 1).

As in experiment 1, we distinguished three groups of stimuli (see figure 4 for examples and table 3 for statistics). Again, the first group $(\mathrm{E}>\mathrm{I})$ was clearly the largest group, with 154 stimuli $(83.7 \%$ of the stimuli), with a mean and median difference in identification of $12.5 \%$ and $46.0 \%$, respectively. The second group $(E=I)$ now consisted of only 19 stimuli $(10.3 \%$ of the stimuli), 18 of which had $0 \%$ identification in both conditions. In the last group $(\mathrm{E}<\mathrm{I})$, with only 11 stimuli $(6.0 \%)$, the I versions were easier to identify than the E versions, and the difference was somewhat larger than in experiment $1($ mean $=-15.3 \%$, median $=-12.0 \%)$. The correlations between the relative area difference and the identification difference (compared to the original silhouette) were lower in this experiment $(r=0.28$ for $\mathrm{E}$ and $r=0.26$ for $\mathrm{I}$; both $p$ s $<0.001$ ).

\section{Experiment 3: Salient curvature singularities}

\subsection{Introduction}

In this experiment, we start again from curvature singularities but, instead of selecting them on the basis of their absolute curvature values, we now ask an independent group of subjects to select the most salient curvature singularities. Because this is probably a more valid selection criterion, we expect the identification rates to increase in this experiment. 


\subsection{Methods}

Only aspects of the methods that are unique to this experiment are described here (see also section 2 and table 1).

5.2.1 Subjects. 206 participants (44 male, 162 female, mean age 19.0 years) were tested in seven sessions with a maximum number of 30 participants in each session.

5.2.2 Stimuli. Before we could create new straight-line versions with empirically derived salient curvature singularities, we needed to run another experiment, asking an independent sample of 394 subjects (also first-year psychology students at the University of Leuven) to select the most salient curvature singularities of each contour from the 260 outlines derived from the Snodgrass and Vanderwart set. The whole set was subdivided into ten groups of 26 stimuli each (matched for several possibly relevant variables; see section 2) and each subject received only 26 stimuli (half in E version and half in I version, with stimulus assignment counterbalanced across subjects). On average, each stimulus was thus presented to 20 subjects per condition. In the E versions, all of the local curvature extrema were shown as small black dots superimposed on the contour, and likewise for all of the inflections in the I versions. A computer program assisted the subjects in selecting the most salient dots superimposed on the contour: a blue cross was always shown on the contour, superimposed on the curvature singularity nearest to the cursor; a point could be selected by clicking the left mouse button, and the small black dot then turned into a larger blue dot; it could be removed from the selection by moving over it again (the blue cross then turned into a red one) and clicking the left mouse button again, etc. The subjects needed to look at the whole contour first for $1 \mathrm{~s}$ before they could begin selecting points. The contour remained on the screen for as long as the subject wished (but at least for $5 \mathrm{~s}$ ); the subjects could indicate that they were finished by pressing the 'ENTER' key, and then all of the selected points were written to a data file.

For all of the stimuli in experiment 3, we selected the curvature extrema and the inflections that were marked by at least 5 subjects from the preceding experiment (this does not guarantee an equal number of extrema and inflections; see table 4). As in all the other experiments, the selected points (either E or I) were then connected by straight-line segments to produce E versions and I versions of all 184 stimuli (see figures 4,7 , and 8 for examples).

5.2.3 Procedure. The total set of 184 stimuli was subdivided into four groups of 46 stimuli each (matched for several possibly relevant variables; see section 2) and each subject received only 46 stimuli (half in E version and half in I version, with stimulus assignment counterbalanced across subjects). On average, each stimulus was thus presented to 26 subjects per condition.

\subsection{Results and discussion}

For the most important results, see tables 2, 3, and 4 and figure 5c. As in experiments 1 and 2, identification was still reasonable to good for the E versions, whereas it was poor for the I versions: mean and median identifications were $60.7 \%$ and $71.7 \%$ for E versions and $20.0 \%$ and $3.8 \%$ for I versions. A $t$-test on the averages revealed a large statistical difference between the two conditions $\left(t_{366}=9.53, p<0.001\right)$. As expected, identification was better for the E versions than in experiments 1 and 2, because the most visually salient extrema were now selected (based on empirically established salience measures). It cannot be due to the mean number of selected extrema, because this number is now lower than in experiments 1 and 2 (24.2 versus 29.7 and 27.4, respectively). It is just a matter of a better selection. For the I versions, the difference was not significant (somewhat larger than in experiment 2 but virtually identical to that in experiment 1). We will return to this issue in section 8 . 
As in experiments 1 and 2, we distinguished three groups of stimuli (see figure 4 for examples and table 3 for statistics). As before, the overwhelming majority of the stimuli $(166$ or $90.2 \%)$ were in the first group $(\mathrm{E}>\mathrm{I})$, with an average and median difference in identification of $45.6 \%$ and $39.9 \%$, respectively. The second group $(E=I)$ now consisted of only 11 stimuli $(6.0 \%$ of the stimuli), 9 of which were not identified at all; the last group $(\mathrm{E}<\mathrm{I})$ now had only 7 stimuli $(3.8 \%)$ and the difference was not big $($ mean $=-11.5 \%$, median $=-10.2 \%$ ). The correlations between the relative area difference and the identification difference (compared to the original silhouette) were $r=0.14$ for $\mathrm{E}(p=0.066)$ and $r=0.33$ for I $(p<0.001)$.

Comparison of the results of the first three experiments reveals a striking pattern: with the improved selection criterion for curvature extrema, the identification rates clearly increase for the E versions (for all comparable points across the whole range: mean, median, first and third quartile - see table 2). The correlation with the area measure clearly decreases (from $>0.50$ in experiment 1 to $<0.15$ in experiment 3 ). For the I versions, the identification rates do not vary so strongly (experiment 2 is somewhat lower) and neither do the correlations with the area measure (against lowest in experiment 2). We will return to this observation in section 8 .

\section{Experiment 4: Salient points and midpoints}

\subsection{Introduction}

In contrast to the preceding three experiments, in which the points to be connected were always mathematically defined curvature singularities (selected according to mathematically defined criteria, as in experiments 1 and 2; or based on empirically defined criteria, as in experiment 3), we now select the points to be connected in a completely empirical way. We have asked another independent sample of 161 subjects to mark salient points along the contours of our 260 stimuli (De Winter and Wagemans 2008; see also De Winter and Wagemans 2004) and we have taken the most popular ones as points of departure for our new straight-line versions.

\subsection{Methods}

Only the aspects of the methods that are unique to this experiment are described here (see also section 2 and table 1).

6.2.1 Subjects. 108 participants (18 male, 90 female, mean age 19.0 years) were tested in four sessions with a maximum number of 30 participants in each session.

6.2.2 Stimuli. Before we could create new straight-line versions with empirically derived salient points, we needed to run another experiment, asking an independent sample of 161 subjects (also first-year psychology students at the University of Leuven) to select the most salient points along each contour from the 260 outlines derived from the Snodgrass and Vanderwart set. The whole set was subdivided into four groups of 65 stimuli each (matched for several possibly relevant variables) and each subject received one set of 65 stimuli. On average, each stimulus was thus presented to 40 subjects per condition. For more details of the data acquisition and data analysis of this study, see De Winter and Wagemans (2008; see also De Winter and Wagemans 2004). In essence, the selection of the most salient points proceeded as follows. First, the raw frequency data were smoothed by a Gaussian function (with an SD of 5 pixels) and then the local maxima from this salience distribution were selected if their value was higher than a particular threshold. Because the contours differed widely in how distributed the salience values were, the threshold was set adaptively. It was determined as the integer value of the mean smoothed salience (eg 7 for mean smoothed salience of 7.93). 
We now have all of the salient points to be connected by straight-line segments in the condition SP100 (for $100 \%$ of the salient points selected according to the procedure described above). In the comparison condition, we used the points half-way inbetween these salient points [measured on the original outline as the Euclidean distance in pixels from point to point and then accumulated along the outline; see also appendix 1 in De Winter and Wagemans (2006)]. We call the straight-line versions connecting these midpoints the MP100 versions. Because we want to know how fast identification decreases when fewer points are connected, we have also created a condition in which the $75 \%$ most salient points of the previously determined salient points are used (SP75 condition). Likewise, we have also determined new midpoints between those $75 \%$ salient points and connected these by straight-line segments to create the MP75 condition (see figures 4,7 , and 8 for examples).

6.2.3 Procedure. The total set of 184 stimuli was subdivided into four groups of 46 stimuli each (matched for several possibly relevant variables; see section 2). Each subject received all four groups of 46 with a counterbalanced assignment of stimuli to conditions across subjects (group A to SP100, B to MP100, C to SP75, D to MP75, for subject 1; group B to SP100, C to MP100, D to SP75, A to MP75, for subject 2; etc). Hence, each subject saw all of the 184 only once and, on average, each stimulus was presented to 27 subjects per condition.

\subsection{Results and discussion}

For the most important results, see tables 2, 3, and 4 and figures $5 \mathrm{~d}$ and $5 \mathrm{e}$. As expected, the mean identification rates for the SP versions were much higher than for the MP versions (68.9\% versus $34.4 \%$, for SP100 and MP100, respectively). The medians differed even more (85.2\% versus $25.9 \%$, respectively). A similar difference was obtained for the $75 \%$ versions $[54.7 \%$ versus $18.0 \%$, for SP75 and MP75, respectively (medians: $59.3 \%$ versus $3.7 \%$, respectively)]. An ANOVA revealed a significant effect of point type (SP versus MP) $\left(F_{1,183}=309.70, p<0.001\right)$, and of the number of points $(100 \%$ versus $75 \%)\left(F_{1,181}=144.03, p<0.001\right)$, but no interaction effect $\left(F_{1,183}<1\right)$. Compared to experiment 2, where each straight-line version was based on the same number of curvature singularities as the present number of salient points, the identification rates are higher here (for all comparable points across the whole range: mean, median, first and third quartile-see table 2). For example, for the difference between the means $t_{366}=4.20, p<0.001$. The same is true for the comparison between inflections and midpoints $\left(t_{366}=6.74, p<0.001\right)$, where this difference is less obvious: the midpoints between selected salient points are by no means guaranteed to be close to inflections (whereas salient points are often close to extrema, see Dr Winter and Wagemans 2008).

The extent to which MP versions are still relatively well-identifiable seems to depend on the distribution of MPs along the contour. By definition, MPs are located midway between salient points and, hence, they are relatively evenly distributed, whereas the location of inflections may be more erratic (especially in experiment 2, where their locations depend on the average absolute curvature of their neighbouring extrema). The likelihood of having an MP version that is similar to an SP version (and thus easier to identify) is higher in the case of a rather complex shape, with a long outline, a large number of points, and relatively short distances between the points. This observation is confirmed by a calculation of these parameters: compared to the $25 \%$ most-difficult-to-identify stimuli, the $25 \%$ easiest-to-identify stimuli have a longer outline (1564 versus 1179 pixels, $t_{45}=3.302, p<0.002$ ), more selected points (36 versus $22, t_{45}=4.416, p<0.001$ ), and a shorter average distance between them ( 35 versus 53 pixels, $t_{45}=5.132, p<0.001$ ). We will return to such object differences in section 8 . 
As in the preceding experiments, we distinguished three groups of stimuli (see figure 4 for examples and table 3 for statistics). As before, the overwhelming majority of the stimuli (163 or $88.6 \%$ ) were in the first group (SP100 > MP100), with a mean and median difference in identification of $39.6 \%$ and $33.0 \%$, respectively. The second group $(\mathrm{SP} 100=\mathrm{MP} 100)$ now consisted of only 14 stimuli $(7.6 \%$ of the stimuli), 6 of which were not identified at all and 5 of which were identified perfectly in both conditions. Finally, the third group (SP100<MP100) had only 7 stimuli $(3.8 \%)$, with a mean and median difference of $-14.8 \%$ and $-15.0 \%$. The same distinction was also made for the $75 \%$ versions. Here too, the majority of the stimuli (152 or $82.6 \%$ ) were in the first group (SP75 > MP75), with a mean and median difference in identification of $44.9 \%$ and $44.4 \%$, respectively. The second group (SP75 = MP75) now consisted of 24 stimuli $(13.0 \%$ of the stimuli), 17 of which were not identified at all. Finally, the third and smallest group (SP75 < MP75) had only 8 stimuli $(4.3 \%)$, with a mean and median difference of $-10.2 \%$ and $-9.3 \%$, respectively.

The correlations between the relative area difference and the identification difference (compared to the original silhouette) were rather low (even negative) for the $100 \%$ versions, $r=-0.09(p=0.360)$ for SP100 and $r=-0.11$ for MP100 $(p=0.150)$, and positive for the $75 \%$ versions but significant only for SP75, $r=0.24(p<0.001)$, not for MP75, $r=0.02(p=0.812)$.

\section{Experiment 5: Midpoints with tangent lines}

\subsection{Introduction}

In all of the experiments so far, the straight-line versions in which inflections (experiments 1, 2, and 3) or midpoints (experiment 4) were connected by straight-line segments were much harder to identify than the corresponding versions connecting extrema or salient points. Despite Lowe's (1985) demonstration and Biederman's (1988) empirical confirmation that the location of the selected points does not matter for Attneave's sleeping cat (compare figure 1c to $1 \mathrm{~b}$ ), this major difference between $\mathrm{E}$ and I versions, or SP and MP versions, should not surprise us. Contour segments around inflections have generally very little curvature and the same tends to be true for segments around midpoints, because they are located far away from the most salient points (usually points with high absolute curvature values, if not curvature extrema). When connecting I or MP by straight-line segments, they become corner points, whereas originally they were located in contour segments where curvature did not change much, far away from strong curvature changes. In other words, removing continuous curvature changes in creating straight-line versions usually implies replacing shallow curvature changes by straightline segments with the same local orientation and replacing strong curvature changes by corners in the same location in the case of E and SP versions. In contrast, shallow curvature changes might become corners and strong curvature changes might be replaced by straight-line segments in the case of I and MP versions, and the original orientation of the contour segments surrounding I or MP is usually changed quite dramatically. In this new experiment, we ask how difficult it is to identify MP versions when no spurious corners are introduced at the original location of the MPs and local contour orientations are preserved at MPs.

\subsection{Methods}

7.2.1 Subjects. 24 participants (4 male, 20 female, mean age 18.8 years) were tested in one session in a room with 24 PCs.

7.2.2 Stimuli. In this experiment, we used only one version of each stimulus. We created new straight-line versions starting from the same midpoints as in experiment 4 . Instead of connecting the selected points by straight-line segments, as we did in all of the preceding experiments, we now fitted a tangent line through each of the midpoints and 
then had new corners where neighbouring tangent lines intersected. We call this new version MPT for midpoint tangents. Some of these MPT versions were problematic because the intersections created X-crossings or the intersection points were sticking out too far. We eliminated these from the stimulus set, retaining 142 stimuli to be tested (see figures 4, 7, and 8 for examples and our website for a complex list of the selected stimuli).

7.2.3 Procedure. All subjects received the 142 stimuli in a new random order, so we have identification rates per stimulus that are based on 24 subjects each.

\subsection{Results and discussion}

For the most important results, see tables 2, 3, and 4 and figure 5f. As expected, the mean identification rate for these new MPT versions was much higher (mean $60.2 \%$, median 62.5\%) than for the MP100 versions from experiment 4 (34.4\% and 25.9\%, respectively) ( $t_{324}=8.37, p<0.001$, between the means), but still somewhat lower than for the SP100 versions from experiment $4(68.9 \%$ and $85.2 \%)\left(t_{324}=2.31, p=0.02\right.$ between the means). Restricting the comparisons to the same 142 stimuli from experiment 4 confirms these trends: identification is clearly better than for the MP100-142 versions $\left(t_{282}=11.4, p<0.001\right)$, and no longer much worse than for the SP100-142 versions $\left(t_{282}=1.50, p=0.13\right)$. In other words, preserving the local orientation around the midpoints, instead of introducing spurious corner points at these locations, removes a great deal of the identification cost. Hence, the fact that corners are located where no strong curvature changes were present in the original outlines has probably contributed a lot to the difficulty in identifying straight-line versions based on I or MP in the previous experiments.

As in the preceding experiments, we distinguished three groups of stimuli, based on the sign of the difference between the present MPT versions and the corresponding SP100-142 versions (see figure 4 for examples and table 3 for statistics). The group with SP100-142 > MPT was still the largest group, containing 73 stimuli $(51.4 \%)$, but it was clearly smaller than in all of the preceding experiments. The average and median difference was also not that big $(22.3 \%$ and $20.8 \%$, respectively). The second largest group (48 stimuli, 33.8\%) now was the group with the reversed difference (SP100-142 < MPT). All of the stimuli in the last group with SP100-142=MPT (21 stimuli, 14.8\%) were either perfectly identified (18) or not at all (3). Examples of each group are given in figure 4.

As for the MP versions in experiment 4, the correlation between the relative area difference and the identification difference (compared to the original silhouette) was very low for the present MPT versions $(r=0.08, p=0.200)$.

\section{General discussion}

The research reported in this paper started from Attneave's (1954) sleeping cat demonstration. It is useful to distinguish between two aspects of this demonstration: first, that continuous curvature along the contour is not important for object identification and, second, that the critical information about shape is located at curvature extrema. We have tested these two aspects by asking a large number of subjects to try to identify a much larger set of better-controlled stimuli. All of our stimuli are modified outlines derived from line drawings of everyday objects (Snodgrass and Vanderwart 1980). They do not contain internal features as did Attneave's drawing of a sleeping cat but that is not an invalidating limitation because we compare the identification rates of the modified stimuli with those of the smoothly curved outline contours, not the original line drawings. It just allows for much better control of where along the contour we select points to be connected by straight-line segments. In doing this, we can examine differences between different ways of selecting points and between different objects. 
The results provide partial support for Attneave's original ideas but also interesting additional insight in how shape is specified by contour information.

Addressing the first aspect of Attneave's demonstration - is it possible to identify objects based on contours consisting of straight-line segments only? - the answer is a balanced yes: identification was still possible in many cases but the variation between stimuli and conditions was very large (between $0 \%$ and $100 \%$ identification in all of the experiments). There are a number of stimuli for which continuous curvature along the contour is not important but for others it does seem to play an important role. Addressing the second aspect of Attneave's demonstration-is the critical information about shape located at curvature extrema? - the answer is again a balanced yes: in all of the experiments, identification was considerably easier when curvature extrema (E) rather than curvature inflections (I) were connected by straight lines. However, in many cases straight-line versions could also be identified when they were connecting other points and, for the straight-line versions connecting curvature extrema, the particular selection of extrema played an important role as well (again, from $0 \%$ to $100 \%$ variation). Moreover, identification was highest when visually salient points (SP, as marked by independent subjects) were connected and these do not necessarily correspond to mathematical curvature extrema (see De Winter and Wagemans 2008). Furthermore, the relative identification cost for straight-line versions connecting inflections or midpoints (MP) between salient points could often be removed by preserving the local orientation around the midpoints, instead of introducing spurious corner points at these locations. In this sense, curvature extrema do not have such a special status as corner points in straight-line versions. A different test of the role of curvature extrema would be to present only points at their locations. We have performed this test as part of our large-scale study on the identification of fragmented outlines (Panis et al 2008).

The strong variation in identifiability between stimuli and conditions cannot be explained by a simple variable. An obvious candidate is the number of points connected by straight-line segments. Indeed, within each experimental condition, there is a significant correlation between the number of corner points in each stimulus and its identification rate (varying between 0.18 and 0.48 ). However, there is also a considerable variation between conditions, which cannot be explained by the number of points. For example, within experiment 1 , the number of points was exactly the same in the E and I conditions and the average identification was $46.4 \%$ versus $20.4 \%$, respectively. Experiments 2 and 4 were matched for number of points but identification appeared easier when salient points or midpoints were connected $(68.9 \%$ versus $34.4 \%$, respectively) than when extrema or inflections were connected ( $53.4 \%$ versus $14.2 \%$, respectively). Improving the criterion for selecting the extrema or salient points from experiments 1 to 4 , making it more perceptually relevant than mathematically strict, had a strongly beneficial influence on identification, although the number of selected points did not increase $(29.7,27.4,24.2,27.6)$. All in all, the correlation between the average number of points and the average identification rate across experiments was low and even slightly negative $(r=-0.06, p=0.847)$, and the same was true for the correlation between the median values $(r=-0.08, p=0.805)$.

Another obvious candidate to explain the decrease in identifiability when going from smoothly curved contours to straight-line versions is the deviation between the stimuli. When the straight-line version approaches the original contour very well, it is logical to expect that identification will not be lower. Across experimental conditions, the correlation between the average size of the stimulus difference (cumulative area of nonoverlapping stimulus parts) and the average identification rate as such was strongly negative $(r=-0.84, p<0.001)$ and the same was true for the medians $(r=-0.88$, $p<0.001)$. So, a small area difference clearly contributes to identifiability. 
Looking at a finer scale within each experimental condition, there was a significant positive correlation between the area difference and the drop in identification in six experimental conditions (varying between 0.24 and 0.53 ). The correlation was highest in the experiments where the selection was probably the least psychologically relevant (one extremum per lob in experiment 1 and the largest absolute curvature in experiment 2). In those experiments, large sections of the original contour are often missing when selecting inappropriate points (see figure 6) and this causes a great variability in identifiability. However, the correlation was quite low (even negative) in five other experimental conditions where the average identification was either rather high (eg experiment 3, E versions, mean identifiability $=60.7 \%, r=0.14$; experiment 4, SP100 versions, mean identifiability $=68.9 \%, r=-0.09$ ) or rather low (eg experiment 4, MP100 and MP75 versions, mean identifiability $=34.4 \%$ and $18.0 \%, r=-0.11$ and 0.02 , respectively).

This means that there is clearly more going on that determines whether the critical shape information for object identification is preserved in the straight-line version of the outline. A small deviation from the original outline (defined as the area of nonoverlap) is by no means a necessary or sufficient condition for identification. We noticed that, in the case of large average area differences (eg bigger pieces being chopped off), the effect of the area difference on identifiability is usually substantial; whereas, in the case of small average area differences (eg missing or deformed details), the effect on identifiability can be small or large, depending on the diagnosticity of the detail. We have quantified this impression by calculating the correlation between the mean area difference for each experimental condition and the size of the correlation between area difference and identification rate in that condition $(r=0.639$, $p=0.034$; and similar but smaller for the medians, $r=0.540, p=0.086$ ).

Let us have a closer look at the stimulus differences to try to understand what the additional factors may be. There is a group of objects that appear quite robust for the transformation from smoothly curved contours to straight-line versions, regardless of the specific selection criterion for the corner points, whereas another group of objects cannot be identified in any of the straight-line versions (see figure 7). Starting from the highly identifiable silhouettes $(>90 \%)$, we sorted the list of objects $(n=117)$ on the average identification rates for all straight-line versions (except SP75, MP75, and MPT). We then distinguished between the top group of 25 objects that remain pretty identifiable (between $95.5 \%$ and $69.5 \%$ ) and the bottom group of 25 objects that become hardly identifiable (between $31.0 \%$ and $5.0 \%$ ). The top group includes mostly objects with relatively complicated shapes or global shape characteristics (not local distinctive features) that are rather unique. This group contains many animals with distinctive global shapes (eg giraffe, deer, seahorse, elephant, bear, ostrich, spider, chicken, pig, swan, rhinoceros, squirrel; see figure 7a) but also other biological objects or artifacts with a characteristic shape (eg anchor, flower, glasses, leaf, carrot, windmill, watering can, kite, helicopter; see figure $7 \mathrm{~b}$ ). The bottom group includes mostly objects with relatively simple shapes (with few curvature singularities); these can be biological (eg lips, lemon, banana, cherry, strawberry; see figure 7c) as well as man-made (eg hat, pan, balloon, shoe, toothbrush, sock, boot; see figure $7 d$ ).

Zooming in on the subgroup for which the selection criterion does play a role may be interesting to try to reveal necessary and sufficient conditions for identification on the basis of straight-line versions. For several objects, identification is still relatively good for straight-line versions based on E or SP but rather poor for those based on I or MP. To obtain these objects in a systematic way, we again started from the highly identifiable silhouettes $(>90 \%)$ and selected those with reasonable average identification for all E and SP100 versions $(>60 \%)$. We then sorted them according to the 


\begin{tabular}{|c|c|c|c|c|c|c|c|c|c|}
\hline \multirow{2}{*}{$\begin{array}{l}\text { Number } \\
\text { and } \\
\text { name }\end{array}$} & \multirow{2}{*}{$\begin{array}{l}\text { Original } \\
\text { silhouette }\end{array}$} & \multicolumn{2}{|c|}{ Experiment 1} & \multicolumn{2}{|c|}{ Experiment 2} & \multicolumn{2}{|c|}{ Experiment 3} & \multicolumn{2}{|c|}{ Experiment 4} \\
\hline & & E & I & $\mathrm{E}$ & I & $\mathrm{E}$ & I & SP100 & MP100 \\
\hline 200 & & & & & & & & & \\
\hline Seahorse & $100 \%$ & $96 \%$ & $25 \%$ & $100 \%$ & $60 \%$ & $96 \%$ & $15 \%$ & $100 \%$ & $93 \%$ \\
\hline
\end{tabular}

(b) 48

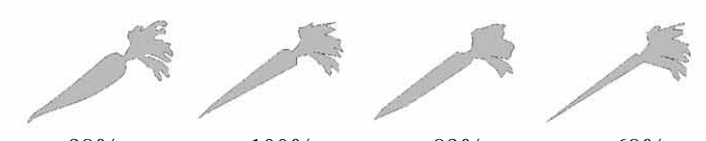

Carrot

$98 \%$

$100 \%$

$89 \%$

$68 \%$
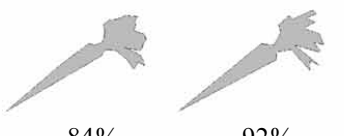

$92 \%$

$70 \%$

$100 \%$

(c)

135
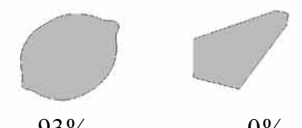

$0 \%$

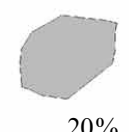

$0 \%$

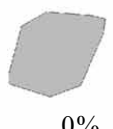

$0 \%$
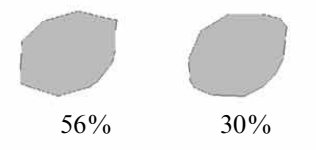

(d)

179
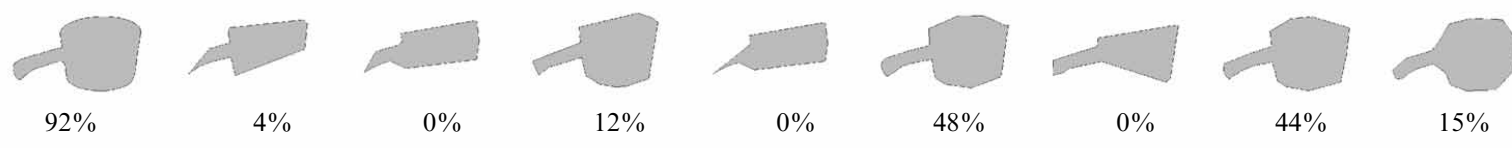

Figure 7. (a) and (b) Examples of straight-line versions that can still be identified in almost all conditions. These are typically objects with a highly distinctive global shape. (c) and (d) Examples of straight-line versions that are difficult to identify in almost all conditions. These are typically objects with simple shapes where large parts are easily lost. The identification rate is mentioned below each stimulus. 


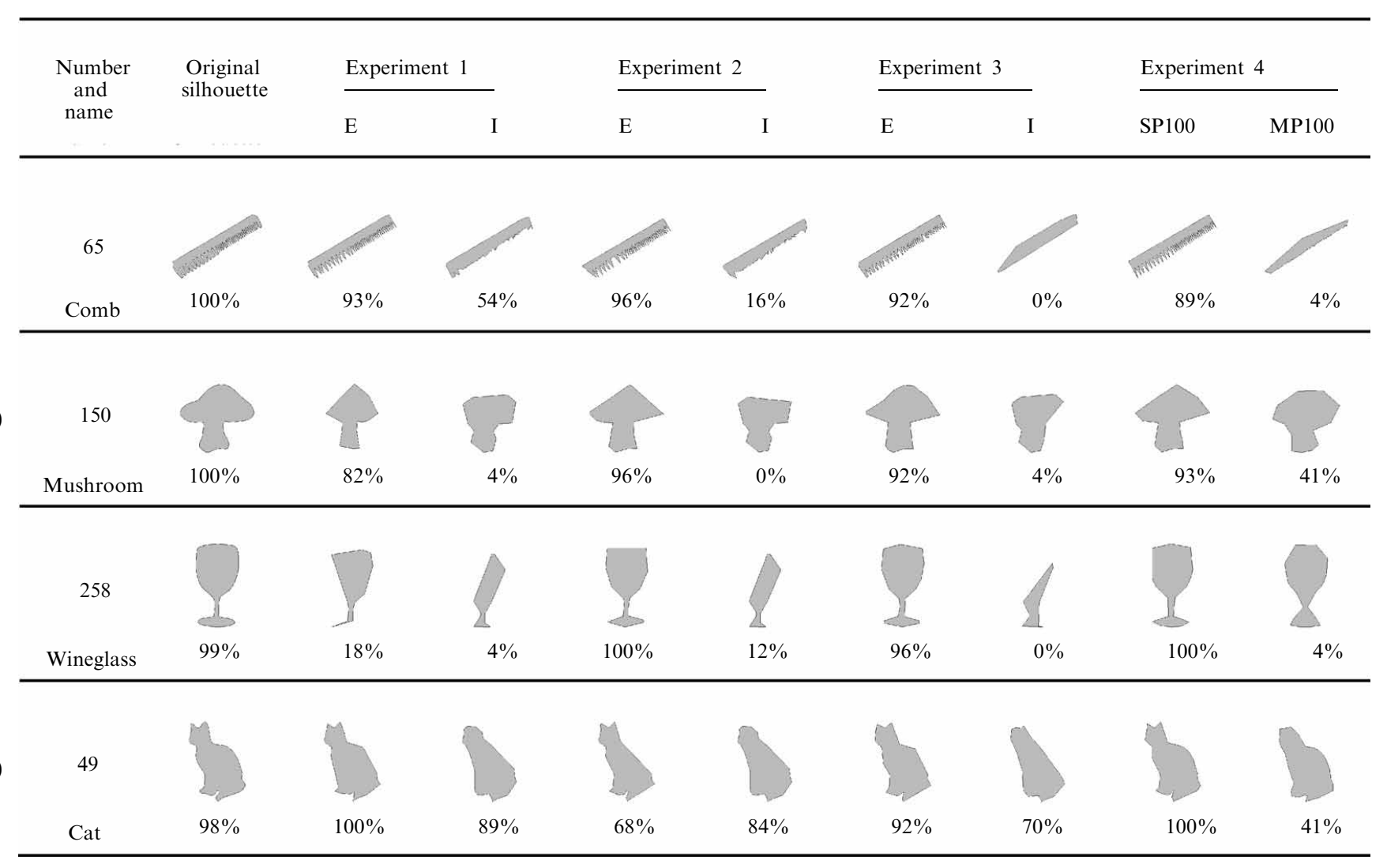

Figure 8. Examples of straight-line versions with a large difference between E/SP versions and I/MP versions. These are usually moderately complex shapes. (a) An example of an object with a considerable degree of curvature variation along the contour but a strikingly repetitive pattern that is preserved in E/SP versions but not in I/MP versions. (b) and (c) Examples of objects with simpler shapes in which the characteristic global properties are preserved in E/SP versions but not in I/MP versions. (d) Our versions of an outline drawing of a cat. The distinctive local features (ears) are preserved in E/SP versions but not in I/MP versions. The identification rate is mentioned below each stimulus. 
size of the difference with the average identification for all I and MP100 versions. The majority of the objects with the biggest difference $(>60 \%)$ have moderately complex shapes (see figure 8 ). Those with a considerable degree of curvature variation in the original silhouette are characterised by a striking regularity such as a frequent repetition of a salient feature (eg comb, pineapple; see figure 8a). in the E/SP version, this is maintained, while it usually disappeared in the I/MP version. Most of the objects in this subset have simpler shapes that happen to be distorted strongly when the corner points are selected badly (eg candle, mushroom, star, lamp, church, duck, pear; see figure $8 \mathrm{~b}$ ). In many of these, the characteristic bilateral mirror symmetry is preserved in the E/SP version but destroyed in the I/MP version (eg dress, wineglass, pants, scissors, hand; see figure 8c). Symmetry is a salient regularity for the visual system (eg Wagemans 1995, 1997) and it has been included as an important factor in theories of shape representation (eg Biederman 1987; Kurbat 1994; Quinlan 1991). Note, however, that symmetry is not a sufficient condition for identification (see figure 8c, 'wineglass' in experiment 4): the global shape characteristics (eg alternation of parts sticking out) must be preserved as well. This corroborates the importance of convexities and concavities in determining an object's part-structure, as proposed in recent work on shape perception (eg Barenholtz et al 2003; Bertamini 2001; Bertamini and Mosca 2004; Cohen et al 2005; Lamote and Wagemans 1999; Vandekerckhove et al 2007).

The more complex shapes (with more curvature variation) within this subgroup for which the selection criterion plays a role, happen to have smaller parts or local features that appear distinctive: when they are preserved in the straight-line version (usually in the E/SP version), the objects can still be identified; however, when they are not maintained (usually in the I/MP version), the objects can no longer be identified. Many of the objects in this subset are animals (eg frog, rabbit, cat, fish, penguin, duck, crocodile, kangaroo; see figure 8d) and the diagnostic features are often typical body parts (eg paws, ears, beak, tail). It is quite striking that this damaging effect can often be repaired by drawing tangent lines through the midpoints (see figure 9). The MPT versions are usually a bit more 'spiky' or 'jerky' and may thus appear more like cartoon versions, but at least their basic shape characteristics are maintained,

\begin{tabular}{llllc}
\hline $\begin{array}{c}\text { Number } \\
\text { and } \\
\text { name }\end{array}$ & $\begin{array}{c}\text { Original } \\
\text { silhouette }\end{array}$ & Experiment 4 & Experiment 5 \\
\cline { 3 - 4 } & & SP100 & MPT \\
\hline Dress & $100 \%$ & $96 \%$ & $0 \%$ & $92 \%$ \\
\hline
\end{tabular}

Frog $100 \%$ 100\%

Figure 9. Examples of straightline versions that were hardly identifiable as I/MP versions but that become identifiable again when spurious corner points at I/MP are avoided by drawing straight lines through them in the MPT versions. The identification rate is mentioned below each stimulus. 
and hence they remain identifiable. This is again in line with recent work on the role of concavities and convexities in an object's part-structure (Barenholtz et al 2003; Bertamini 2001; Bertamini and Mosca 2004; Cohen et al 2005; Lamote and Wagemans 1999; Vandekerckhove et al 2007). Despite substantial differences regarding the role of different curvature singularities, similar differences between different object classes (depending on their global shape characteristics) were obtained in the study of identification of fragmented outlines (Panis et al 2008).

In summary, Attneave (1954) was right in pointing out that continuous curvature along the contours is often not necessary for identification. However, it is slightly misleading to argue on the basis of the sleeping cat demonstration (see figure 1b) that curvature extrema contain most of the information about the contour because connecting them with straight-line segments preserves identifiability. First, for sufficiently complex objects without critical distinctive features at the level of small details, it does not matter much which points are selected to be connected by straight-line segments (see also figure 1c): they can be identified by their global shape which is preserved regardless of the points being connected. Second, for relatively simple shapes with little curvature variation (eg banana) or relatively few, rather large round sections (eg apple), connecting only the global or largest curvature extrema by straight-line segments will never retain the basic shape characteristics. Third, for the large majority of objects, mostly with intermediate levels of complexity, the specific selection of points will determine whether identification is still possible in the straight-line version: when the global shape characteristics (eg repetition or mirror reflection of part structure) or distinctive local features (eg typical body parts) are preserved, identification is good and otherwise it is poor. Having a large and reliable benchmark data set with identification rates of a large number of everyday objects in different straight-line versions will be useful to test future more specific proposals about critical shape information in outline contours.

Acknowledgments. This research was supported by a research grant from the University Research Council (OT/00/007) and from the Research Foundation (FWO-Vlaanderen G.0189.02) to JW. We would like to thank two anonymous reviewers for helpful comments on the previous draft and Sven Panis for assistance with the data collection and for interesting discussions. After the first submission of the manuscript, Joeri De Winter has died in a tragic accident.

\section{References}

Attneave F, 1954 "Some informational aspects of visual perception" Psychological Review 61 $183-193$

Barenholtz E, Cohen E H, Feldman J, Singh M, 2003 "Detection of change in shape: an advantage for concavities" Cognition 89 1-9

Bertamini M, 2001 "The importance of being convex: an advantage for convexity when judging position" Perception $301295-1310$

Bertamini M, Mosca F, 2004 "Early computation of contour curvature and part structure: evidence from holes" Perception 33 35-48

Biederman I, 1987 "Recognition-by-components: A theory of human image understanding" Psychological Review 94115 - 147

Biederman I, 1988 "Aspects and extensions of a theory of human image understanding", in Computational Processes in Human Vision: An Interdisciplinary Perspective Ed. Z W Pylyshyn (Norwood, NJ: Ablex) pp 370-428

Cohen E H, Barenholtz E, Singh M, Feldman J, 2005 "What change detection tells us about the visual representation of shape" Journal of Vision 5313 - 321

De Winter J, Wagemans J, 2004 "Contour-based object identification and segmentation: Stimuli, norms and data, and software tools" Behavior Research Methods, Instruments, and Computers 36 604-624

De Winter J, Wagemans J, 2006 "Segmentation of object outlines into parts: A large-scale integrative study" Cognition $99275-325$

De Winter J, Wagemans J, 2008 "Perceptual saliency of points along the contour of everyday objects: a large-scale study" Perception \& Psychophysics 70 50-64 
Deregowski J B, 1986 "Shape and contour: Dots and dashes" Perception 15217

Feldman J, Singh M, 2005 "Information along contours and object boundaries" Psychological Review $112243-252$

Halverson J, 1992 "The first pictures: perceptual foundations of Paleolithic art" Perception 21 $389-404$

Hoffman D D, Richards W A, 1984 "Parts of recognition" Cognition 18 65-96

Kennedy J M, Domander R, 1985 "Shape and contour: The points of maximum change are least useful for recognition" Perception $14367-370$

Koenderink J J, van Doorn A J, 1982 "The shape of smooth objects and the way contours end" Perception 11 129-137

Kurbat M A, 1994 "Structural description theories: is RBC/JIM a general-purpose theory of human entry-level object recognition?" Perception 23 1339-1369

Lamote C, Wagemans J, 1999 "Rapid integration of contour fragments: From simple filling-in to parts-based shape description" Visual Cognition 6345 - 361

Lowe D G, 1985 Perceptual Organization and Visual Recognition PhD Thesis, Department of Computer Science, Stanford University (Boston, MA: Kluwer)

Lowe D G, 1988 "Organization of smooth image curves at multiple scales" Proceedings of the Second International Conference on Computer Vision, Tampa, FL (IEEE Computer Society Press) pp $558-567$

Norman J F, Phillips F, Ross H E, 2001 "Information concentration along the boundary contours of naturally shaped solid objects" Perception $301285-1294$

Panis S, De Winter J, Vandekerckhove J, Wagemans J, 2008 "Identification of everyday objects on the basis of fragmented outline versions" Perception 37 271-289

Quinlan P T, 1991 "Differing approaches to two-dimensional shape recognition" Psychological Bulletin $109224-241$

Resnikoff H L, 1985 The Illusion of Reality (New York: Springer)

Severens E, Van Lommel S, Ratinckx E, Hartsuiker R J, 2005 "Timed picture naming norms for 590 pictures in Dutch" Acta Psychologica 119 159-187

Snodgrass J G, Vanderwart M, 1980 "A standardized set of 260 pictures: Norms for name agreement, image agreement, familiarity, and visual complexity" Journal of Experimental Psychology: Human Learning and Memory 6 174-215

Vandekerckhove J, Panis S, Wagemans J, 2007 "The concavity effect is a compound of local and global effects" Perception \& Psychophysics $691253-1260$

Van Gool L, Moons T, Pauwels E, Wagemans J, 1994 "Invariance from the Euclidean geometer's perspective" Perception $23547-561$

Wagemans J, 1995 "Detection of visual symmetries" Spatial Vision 9 9-32

Wagemans J, 1997 "Characteristics and models of human symmetry detection" Trends in Cognitive Sciences $1346-352$

Wagemans J, De Winter J, Op de Beeck H, Ploeger A, Beckers T, Vanroose P, 2008 "Identification of everyday objects on the basis of silhouette and outline versions" Perception 37 207-244

Wagemans J, Notebaert W, Boucart M, 1998 "Lorazepam but not diazepam impairs identification of pictures on the basis of specific contour fragments" Psychopharmacology 138 326-333

Witkin A P, 1986 "Scale space filtering", in From Pixels to Predicates: Recent Advances in Computational and Robotic Vision Ed. A P Pentland (Norwood, NJ: Ablex) pp 5-19 


\section{PERTEPTION}

VOLUME 372008

www.perceptionweb.com

Conditions of use. This article may be downloaded from the Perception website for personal research by members of subscribing organisations. Authors are entitled to distribute their own article (in printed form or by e-mail) to up to 50 people. This PDF may not be placed on any website (or other online distribution system) without permission of the publisher. 